\title{
Practices and applications in ambient and intelligent information systems
}

\author{
Quan Z. Sheng ${ }^{1}$ - Wei Emma Zhang ${ }^{1} \cdot$ Elhadi Shakshuki $^{2}$
}

Published online: 5 July 2017

(C) Springer-Verlag London Ltd. 2017

\section{Introduction}

Ambient systems refer to electronic systems that are sensitive and responsive to the presence of people [1]. The general aim of ambient systems is to provide an environment wherein computing devices will become invisibly embedded into the environment and interact with their environment in a seamless, trustworthy, and natural manner [2]. Ambient intelligence system research builds upon advances in sensors and sensor networks, the Internet of Things (IoT), pervasive computing, and artificial intelligence and have experienced tremendous growth in the last few years [3,4].

Despite the advances, ambient systems still pose many new unsolved research challenges due to the open and unreliable nature of the environment and the complexity and heterogeneity of the computing devices and the underlying communication networks. More importantly, novel technologies should address the tasks to develop easy to use and simple to experience products and services [2,5]. For example, how to design transportation planning from the heterogeneous trip data and largely reduce the cost of

Quan Z. Sheng

michael.sheng@mq.edu.au

Wei Emma Zhang

w.zhang@mq.edu.au

Elhadi Shakshuki

elhadi.shakshuki@acadiau.ca

1 Department of Computing, Macquarie University, Sydney, NSW, 2109, Australia

2 Jodrey School of Computer Science, Acadia University, Wolfville, Nova Scotia B4P 2R6, Canada individual people? How to efficiently consume energy in a sharing manner by leveraging information provided by energy provider as well as consumers? How to securely perform purchasing using data from both banking systems and personal accounts but in an efficient manner? Providing solutions to these issues may release the full potential of ambient intelligence system and bring genuine user expectation and satisfaction.

\section{In this special issue}

The papers in this special issue are based on the best papers from the 7th International Conference on Ambient Systems, Networks and Technologies (ANT 2016), which was held at Madrid, Spain, May 23-26, 2016. The conference attracted a good number of scientific papers that contributed to the state-of-the-art in the areas of ambient and intelligent information systems. All the papers invited for this special issue have undergone two rounds of rigorous review process. Based on the reviewers' feedback, 7 papers were selected for publication from 12 invited submissions.

The first two papers deal with the problems of transportation planning and traffic operations. The paper by Usman, et al. entitled "Mobile value of time for trip chains using sigmoid utility" proposes to model value of time for trip chains as regression problem and optimizes the duration of all activities in the schedule to fulfill the constraints of maximization of time utilization and the minimization of the total travel cost. In "Introduction of car sharing into existing car fleets in microscopic travel demand modelling," Heinrichs, et al. present an integrated approach to model car sharing as a new mode for transport within a travel demand model using disaggregated car fleets with car-specific attributes. The approach extends an multinomial logit (MNL) model to 
implement the model choice and allows for the analysis of particular effects on different costs and access restrictions.

The next two papers focus on the issue of power consumption optimization. In "Delay-Aware Power Optimization Model for Mobile Edge Computing Systems," Jararweh, et al. deal with the problem of optimizing power consumption of Mobile Edge Computing (MEC) systems while adhering to delay and capacity constraints. They model the problem as a mixed-integer linear programming (MILP) optimization task and test the proposed model in several real-world scenarios. In "From Competitive Sensor Redundancy to Competitive Service Redundancy in a Smart City Context," Jabeur, et al. present an approach for the detection and relocation of redundant sensors in wireless sensor networks (WSN), particularly in the context of charging electrical vehicles in a smart city. The ultimate goal is to provide the right charging service, at the right location, at the right time, to the right electrical vehicle.

The paper by Shemshadi, et al. entitled "Searching for the Internet of Things: where it is and what it looks like" presents the first crawler engine to identify IoT data sources over the Web and collects large-scale real-world IoT data. In-depth analytical investigation on real IoT data is provided as well as several intriguing findings. In "Technical Feasibility of Context-Aware Passive Payment Authorization for Physical Points of Sale," Wójtowicz, et al. propose a system architecture that enables passive device-less payments. The core element of the system is the context-based risk and trust assessment which allows for dynamic selection of payment authorization methods that constitutes accurate trade-off between security and convenience. Finally, in "Detect Performance Anomalies in Large-Scale Software Systems Using Entropy," Malik, et al. propose to leverage Shannon entropy to measure the uncertainty in detecting the performance anomalies in large-scale systems (LSSs). The goal is to assist practitioners/operators of LSSs to quickly detect both system-wide and localized subsystem anomalies.

The papers included in this special issue cover several important topics and present some of the key directions in this vibrant and rapidly expanding area of research and development. We hope that the set of selected papers provides the community with a better understanding of the current directions and areas to focus in future and inspires your own work.

Acknowledgements The guest editors would like to take this opportunity to thank all the authors for the efforts they put in the preparation of their manuscripts and for their valuable contributions. We wish to express our deepest gratitude to the referees who provided very useful and thoughtful feedback to the authors. Finally, our sincere thanks go to the Editor-in-Chief, Professor Peter Thomas, of Personal and Ubiquitous Computing for his kind support, advice, and encouragements throughout the preparation of this special issue.

\section{References}

1. Aarts E, Harwig R, Schuurmans M (2002) The invisible future. In: Ambient intelligence. McGraw-Hill, Inc., New York, pp 235250

2. Aarts EHL, de Ruyter BER (2009) New research perspectives on ambient intelligence. J Ambient Intell Smart Environ 1(1):5-14

3. Cook DJ, Augusto JC, Vikramaditya RJ (2009) Ambient intelligence: technologies, applications, and opportunities. Pervasive Mob Comput 5(4):277-298

4. Qin Y, Sheng QZ, Falkner NJG, Dustdar S, Wang H, Vasilakos AV (2016) When things matter: a survey on data-centric internet of things. J Netw Comput Appl 64:137-153

5. Sheng QZ, Qin Y, Yao L, Benatallah B (eds.) (2017) Managing the web of things: linking the real world to the web., Morgan Kaufmann 